\title{
Integrated mitochondrial function and cancer- related fatigue in men with prostate cancer undergoing radiation therapy
}

This article was published in the following Dove Press journal:

Cancer Management and Research

\section{Chao-Pin Hsiao' \\ Mei-Kuang Chen² \\ Barbara Daly' \\ Charles Hoppel ${ }^{3}$}

'Frances Payne Bolton School of Nursing, Case Western Reserve University, Cleveland, OH, USA;

${ }^{2}$ Department of Psychology, University

of Arizona, Tucson, AZ, USA;

${ }^{3}$ Center for Mitochondrial Disease, Department of Pharmacology, School of Medicine, Case Western Reserve University, Cleveland, $\mathrm{OH}$, USA
Correspondence: Chao-Pin Hsiao

Frances Payne Bolton School of Nursing, Case Western Reserve University, 2120 Cornell Road, Cleveland, OH 44I064904, USA

$\mathrm{Tel}+\mathrm{I} 2163683343$

Fax + I 2163683542

Email cxh416@case.edu
Introduction: Fatigue experienced by cancer patients is one of the most common symptoms with the greatest adverse effect on quality of life, but arguably the least understood. The purpose of this study was to explore changes in integrated mitochondrial function and fatigue in nonmetastatic prostate cancer patients receiving localized radiation therapy (XRT).

Materials and methods: We proposed a mitochondrial bioenergetics mechanism of radiationinduced fatigue linking impaired oxidative phosphorylation (OXPHOS) through complex III and decreased adenosine triphosphate (ATP) production as consequences of XRT. Integrated mitochondrial function was measured as mitochondrial OXPHOS from patients' peripheral blood mononuclear cells. Fatigue was measured using the revised Piper Fatigue Scale. Data were collected before (day 0) and at day 21 of XRT.

Results: At day 21 of XRT, fatigue symptom intensified in 15 prostate cancer patients $(P<0.05)$. Mitochondrial OXPHOS complex III-linked and uncoupled complex III rates were significantly decreased in mononuclear cells at day 21 during XRT compared to that before XRT $(P<0.05)$. Additionally, increased fatigue appeared to be associated with decreased OXPHOS complex III-linked respiration in patients undergoing XRT.

Conclusion: Fatigue was associated with OXPHOS complex III-linked oxidation and a defect in oxidation starting at complex III in mononuclear cell mitochondria was revealed at day 21 of XRT in 15 prostate cancer patients. Complex III is a potential target for pharmacological and, in particular, nutraceutical interventions, eg, Q10, for design of interventions for CRF.

Keywords: integrated mitochondrial function, oxidative phosphorylation, cancer-related fatigue, radiation therapy, prostate cancer

\section{Introduction}

Prostate cancer is the most common malignancy and the second leading cause of cancer mortality among American men. ${ }^{1,2}$ Localized radiation therapy (XRT) using an intensity-modulated radiation technique is a standard treatment option for men with non-metastatic prostate cancer. ${ }^{3}$ Patients receiving XRT often experience different side effects including fatigue, nausea, vomiting, diarrhea, peripheral neuropathy, and cognitive function impairment that reduce the efficacy of treatment. ${ }^{4}$ Although XRT has increased survival rates for men with prostate cancer, it results in numerous side effects such as urinary and bowel problems, sexual dysfunction, and distressing fatigue during treatment, which persist even in disease-free states. ${ }^{5-7}$

Fatigue is the most prevalent, debilitating, and persistent symptom experienced by patients with cancer. ${ }^{8-10}$ The severity of fatigue reported by men with prostate cancer 
increases significantly during the course of treatments, peaking at the midpoint of treatment, either staying elevated or slightly declining after completion. ${ }^{11,12}$ Cancer-related fatigue (CRF) is defined by the National Comprehensive Cancer Network (NCCN) as a distressing, persistent sense of tiredness or exhaustion. ${ }^{13}$ Of all disease and treatment-related symptoms in cancer, fatigue is one of the most burdensome symptoms with the greatest adverse effect on quality of life, but arguably the least understood. ${ }^{5,14}$

CRF is associated with depression, impaired cognitive function, sleep disturbance, and decreased health-related quality of life. ${ }^{15-17}$ Although many mechanisms have been proposed for $\mathrm{CRF},{ }^{18-22}$ the cause remains elusive. There is no optimal pharmacologic therapy for fatigue. The NCCN Practice Guidelines in Oncology for CRF currently recommends five non-pharmacological interventions, which are activity enhancement, psychosocial improvement, attentionrestoring therapy, nutrition, and sleep. ${ }^{13}$ For pharmacologic interventions, the NCCN guidelines recommend that after ruling out other causes of fatigue, the use of psychostimulants should be considered. One of these is methylphenidate, which showed conflicting results in improving fatigue in two small, randomized, clinical trials. ${ }^{13,23}$ With limited available options to address CRF, novel strategies are needed to identify effective interventions. To guide the development of effective therapies for the management of CRF, identification of the pathophysiological mechanism and related-biomarkers associated with CRF is an essential step.

We have determined that there is a significant relationship between radiation-related fatigue and the differential expression of genes related to mitochondrial biogenesis and bioenergetics. ${ }^{24,25}$ A mitochondrial bioenergetics mechanism of radiation-induced fatigue linking impaired oxidative phosphorylation through complex III has been proposed based on our preliminary data. ${ }^{26}$ Oxidative phosphorylation (OXPHOS) is the process in which adenosine triphosphate (ATP) is formed as a result of the transfer of electrons from nicotinamide adenine dinucleotide (reduced form) (NADH) or flavin adenine dinucleotide (reduced form) $\left(\mathrm{FADH}_{2}\right)$ to oxygen $\left(\mathrm{O}_{2}\right)$ by a series of electron carriers. Mitochondrial OXPHOS is a pivotal system, defined as the oxidation-reduction of fuel molecules by oxygen and the concomitant transduction of this energy into ATP. ${ }^{27}$ Integrated mitochondrial function is measured as OXPHOS and involves transport of substrates into the mitochondria, the generation of reducing equivalents $\left(\mathrm{NADH}\right.$ and $\mathrm{FADH}_{2}$ ) by specific dehydrogenases, entry into the electron transport chain (ETC), leading to coupling to the production of ATP. ${ }^{28,29}$
However, there are no studies investigating integrated mitochondrial function in mononuclear cells and CRF in cancer patients undergoing XRT. Our study explored integrated mitochondrial function and cancer-related fatigue symptom, and determined changes of mitochondrial OXPHOS in peripheral blood mononuclear cells in men receiving XRT for non-metastatic prostate cancer. We found a defect in mitochondrial respiratory complex III, which appeared to be associated with fatigue in patients undergoing XRT.

\section{Material and methods}

\section{Study design, sample, and recruitment}

This was a prospective, exploratory, and repeated measures design. The study was reviewed and approved by both the Institutional Review Board of the Case Comprehensive Cancer Center and the University Hospitals Cleveland Medical Center. The study sample was recruited from a population of patients with localized prostate cancer. Men with nonmetastatic prostate cancer scheduled to receive XRT were enrolled. We excluded patients from the study if they had progressive disease causing significant fatigue; documented major psychiatric illness within 5 years; had uncorrected hypothyroidism or untreated anemia; took sedatives, steroids or nonsteroidal anti-inflammatory agents; or had a second malignancy or a mitochondrial disease.

All participants obtained a verbal explanation, in terms suited to their comprehension, of the study's purpose, the extent of their involvement, confidentiality policies, benefits and potential risks, and right to withdraw from the study. After obtaining written informed consent, demographic information and medical history were obtained via interview and medical record review. This study was conducted in accordance with the Declaration of Helsinki. Study variables included fatigue symptom and integrated mitochondrial function. Peripheral blood samples and questionnaires were obtained from each participant at day 0 (before XRT) and day 21 of XRT. The enrolled study sample received a daily dose of 1.8-2 Gy XRT for 15 total treatments over 21 days.

\section{Patient-reported and clinical variables}

Clinical and demographic characteristics of study participants, eg, age, ethnicity, stage of disease, prostate specific antigen (PSA), clinical stage, and Gleason score, were retrieved by chart review. Fatigue was measured by the validated revised Piper Fatigue Scale (rPFS), which is a 22-item paper-pencil, self-administered questionnaire that measures four dimensions of fatigue (behavioral/severity, sensory, cognitive/mood, and affective) using a $0-10$-point intensity 
rating scale $\left(0=\right.$ none; $10=$ worst intensity).$^{30}$ The rPFS has good reliability and validity with internal consistency ranging from 0.7 to 0.9 across four dimensions in previous studies of patients with cancer. It can be completed in 10 minutes and is relatively easy for subjects to complete.

The Hamilton Depression Rating Scale (HAM-D) was used to screen for depressive symptoms in each subject at each time point. HAM-D is a 21 -item, clinician-rated paper questionnaire with good internal reliability ( $\alpha=0.81-0.98)$. The pre-defined cut-off score for depression is 15 in cancer patients, with higher scores indicating more symptoms of depression. ${ }^{31}$

\section{Peripheral blood mononuclear cells harvest}

Blood samples $(20 \mathrm{~mL})$ were collected using ethylenediaminetetraacetic acid (EDTA) vacutainers, stored at room temperature, and processed within 24 hours. Blood was diluted with an equal amount of phosphate-buffered saline (PBS), layered over a density gradient, Lymphoprep ${ }^{\mathrm{TM}}$ (Accurate Chemical \& Scientifix Corp., Cat. \# 1001967, Westbury, NY, USA), and centrifuged according to the manufacturer's guide. Following centrifugation, the mononuclear cells (buffy coat/cloudy layer) were removed from the interface between Lymphoprep $^{\mathrm{TM}}$ and plasma using a pipettor. Mononuclear cells were collected by centrifugation for 10 minutes and then washed twice with PBS. To lyse red blood cells, the mononuclear cells were resuspended in hypotonic sodium chloride $(0.2 \% \mathrm{NaCl})$ for 1 minute followed by hypertonic sodium chloride $(1.6 \% \mathrm{NaCl})$ for another minute before the last wash with PBS and centrifugation. Standard laboratory procedure and optimal protocol for human peripheral blood mononuclear cells harvest has been published previously. ${ }^{32}$

To prepare samples for mitochondrial OXPHOS, the mononuclear cell pellet was suspended with pre-warmed $\left(37^{\circ} \mathrm{C}\right)$ mitochondria respiration medium (MiR05, $110 \mathrm{mM}$ sucrose, $60 \mathrm{mM}$ potassium lactobionate, $0.5 \mathrm{mM}$ EGTA, 3 $\mathrm{mM} \mathrm{MgCl} \cdot 6 \mathrm{H}_{2} \mathrm{O}, 20 \mathrm{mM}$ taurine, $10 \mathrm{mM} \mathrm{KH}_{2} \mathrm{PO}_{4}, 20 \mathrm{mM}$ HEPES, and $2 \mathrm{mg} / \mathrm{mL} \mathrm{BSA}, \mathrm{pH}=7.1) .{ }^{33}$ The number of live mononuclear cells was counted using a hemocytometer with trypan blue and adjusted to a final concentration of 1.5-2 million cells/mL with MiR05.

\section{Integrated mitochondrial function}

Integrated mitochondrial function was measured as OXPHOS (oxidative phosphorylation) proposed by Puchowicz et al, ${ }^{34}$ involving transport of substrates into the mitochondria, the generation of reducing equivalents by specific dehydrogenases, entry into ETC (electron transport chain), and coupling to ATP synthesis. Mononuclear cell mitochondrial OXPHOS was determined employing a high-resolution respirometer, Oxygraph-2 K using DatLab 4 software (Oroboros Instruments, Innsbruck, Austria). Two published substrateuncoupler-inhibitor protocols were used to determine the respiration rate of complex I, II, III, and IV, as well as fatty acid oxidation. ${ }^{32,33,35}$ As representative samples, we presented procedures of substrate-uncoupler-inhibitor tracing protocol 1 and protocol 2 with final concentrations in Figures 1 and 2.

We measured mitochondrial OXPHOS starting with intact cellular respiration after air calibration of the $\mathrm{O} 2 \mathrm{k}$ system without any additions. As shown in Figures 1 and 2, before permeabilization of mononuclear cells, in protocol 1 the rate of intact cellular respiration following the addition of the exogenous substrates, malate $(\mathrm{m})$ and pyruvate $(\mathrm{p})$, was not changed, represented as intact cell respiration $+(\mathrm{m}+\mathrm{p})$. In protocol 2 , intact cellular respiration $+(m+$ pal $)$ was measured following the addition of the exogenous substrates, malate and palmitoylcarnitine. To access mitochondria, we used digitonin at a final concentration of $20 \mu \mathrm{g} / \mathrm{mL}$ to permeabilize the plasma membrane while maintaining the integrity of the mitochondrial outer membrane of isolated mononuclear cells. ${ }^{35,36}$ Then we added adenosine diphosphate (ADP) to obtain ADP-stimulated respiration. OXPHOS complex I-linked respiration (coupled CI-linked) was measured as ADP-stimulated respiration in the presence of malate, pyruvate, and glutamate. OXPHOS complex I+II-linked respiration (coupled CI + II-linked) was determined as ADPstimulated respiration in the presence of malate, pyruvate, glutamate, and succinate as substrates for complex I and II.

Maximal oxidative capacity was measured after the addition of FCCP, an uncoupler. Next, rotenone was added to inhibit complex I; the resulting rate was considered the rotenone-insensitive rate. The rotenone-sensitive rate was uncoupled complex I activity, which was the difference between maximal oxidative capacity and the rotenone-insensitive rate. Then, we added antimycin A, an inhibitor of complex III, so the difference between the rotenone-insensitive rate and antimycin A-inhibited rate was uncoupled complex II activity. To evaluate uncoupled complex IV, we added tetramethyl-p-phenylenediamine (TMPD) and ascorbate to reduce endogenous cytochrome $c$; the rate was measured, following the injection of sodium azide to inhibit complex IV.

In protocol 2, we measured the fatty acid oxidation rate as the ADP-stimulated respiration in the presence of malate and palmitoylcarnitine, a long-chain fatty acid. The oxidation rate of OXPHOS-coupled complex III-linked was determined 


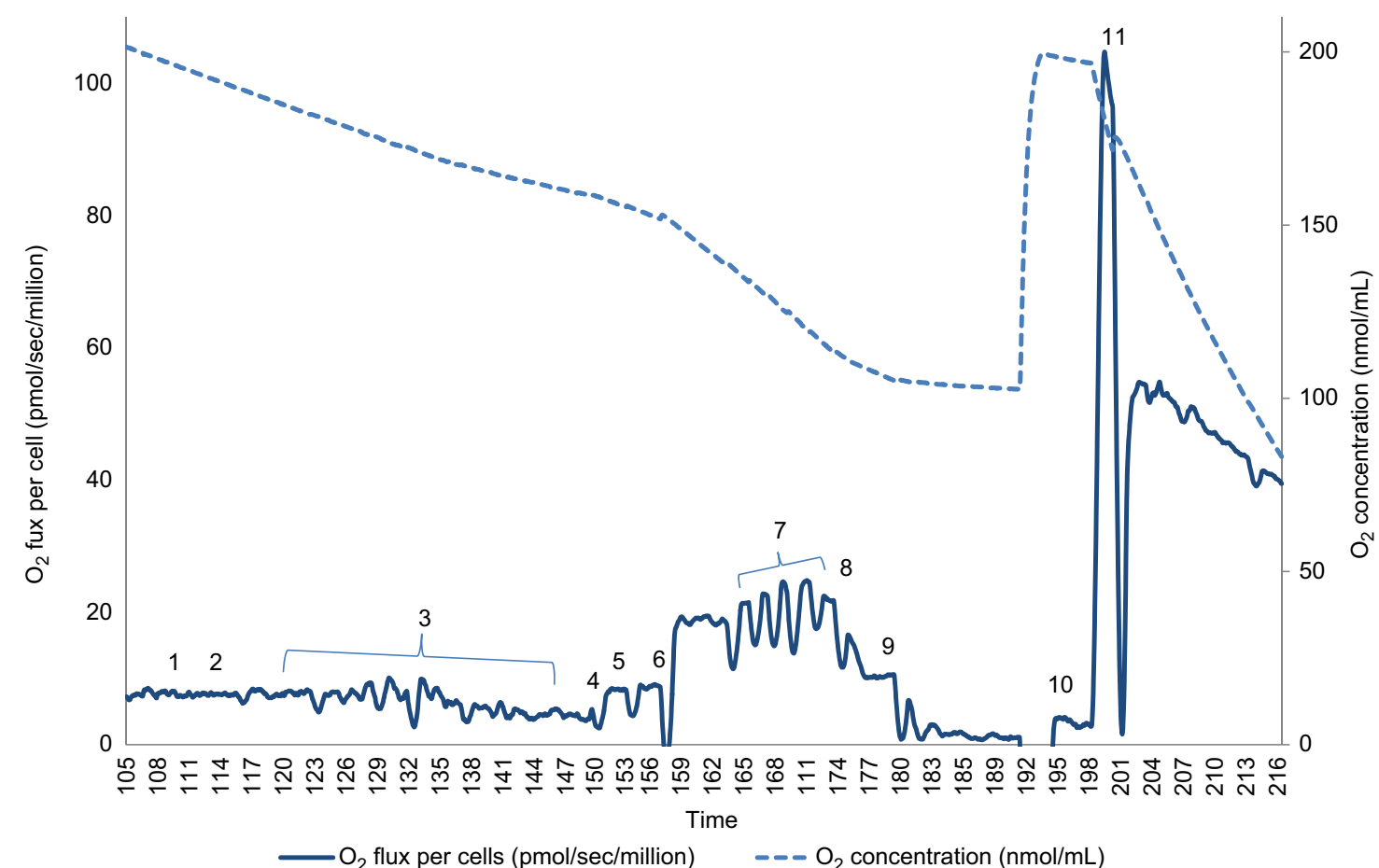

B Substrate-inhibitor tracing protocol 2 in human peripheral blood mononuclear cell

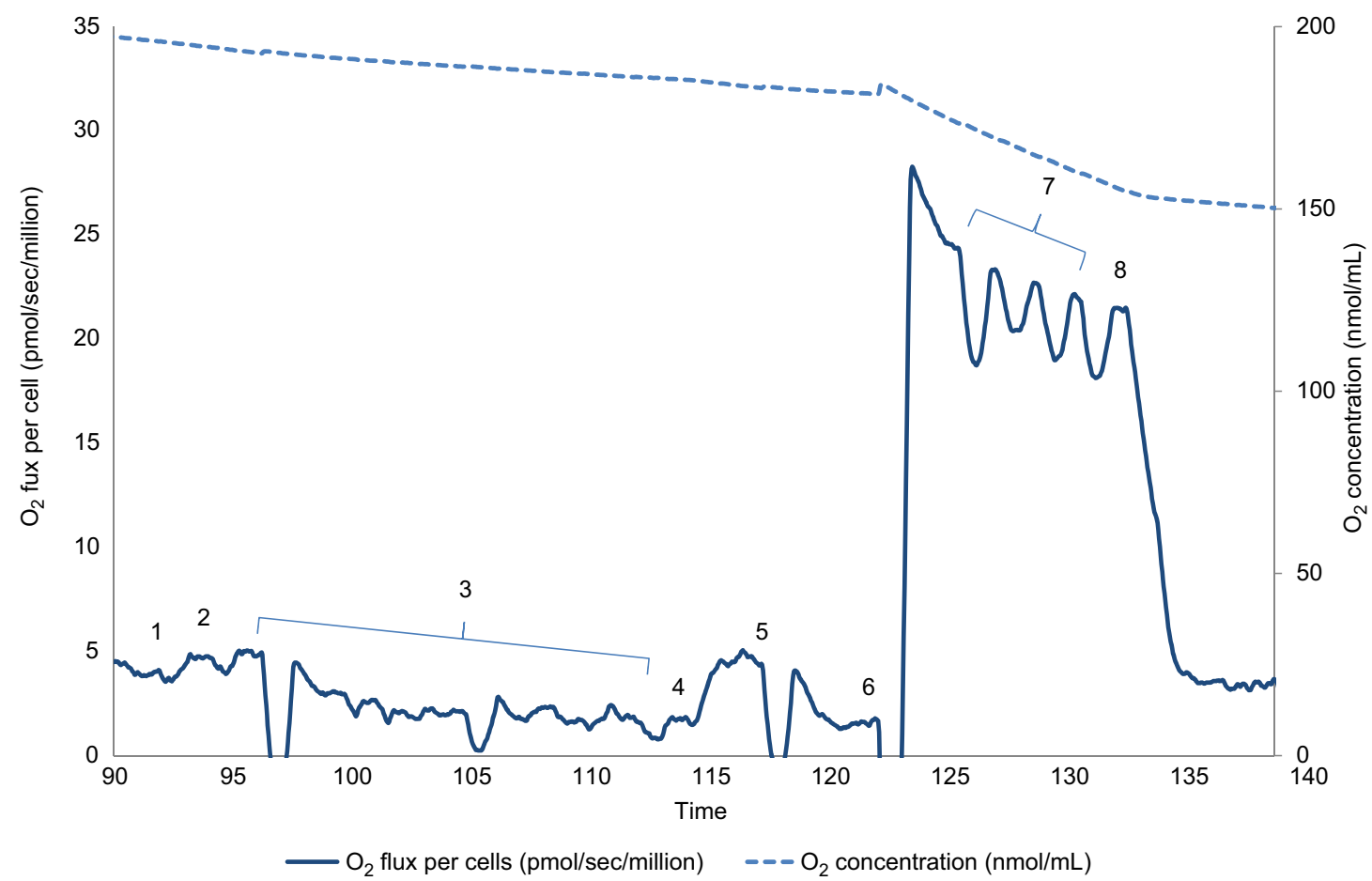

Figure I Substrate-inhibitor tracing of protocols I and 2 in peripheral blood mononuclear cells.

Notes: (A) The oxygen consumption rate is plotted on the left $y$ axis and the oxygen concentration curve is plotted on the right $y$ axis. A total of II additions of reagents and inhibitors are tagged with a numeric symbol. Eleven additions of reagents and inhibitors are tagged with a numeric symbol. I=2 mM malate; $2=2.5 \mathrm{mM}$ pyruvate; $3=20$ $\mu \mathrm{g} / \mathrm{mL}$ digitonin; 4=2.5 mM ADP (adenosine diphosphate); $5=10 \mathrm{mM}$ glutamate; $6=10 \mathrm{mM}$ succinate; 7=0.25 $\mu$ M increment of FCCP (carbonylcyanide-p-trifluoromethoxy phenylhydrazone); 8=150 nM rotenone; 9=125 nM antimycin A; 10=I mM TMPD (tetramethyl-p-phenylenediamine)+2 $\mathrm{mM}$ ascorbate; II=200 mM sodium azide. (B) The oxygen consumption rate is plotted on the left $y$ axis and the oxygen concentration curve is plotted on the right $y$ axis. Eight additions of reagents and inhibitors are tagged with a numeric symbol. I=2 mM malate; $2=10 \mu M$ palmitoylcarnitine; $3=20 \mu \mathrm{g} / \mathrm{mL}$ digitonin; $4=2.5 \mathrm{mM}$ ADP (adenosine diphosphate); $5=150 \mathrm{nM}$ rotenone; $6=0.5 \mathrm{mM}$ DHQ (duroquinol); $7=0.25 \mu \mathrm{M}$ increment of FCCP (carbonylcyanide-p-trifluoromethoxyphenylhydrazone); 8=125 nM antimycin A. 


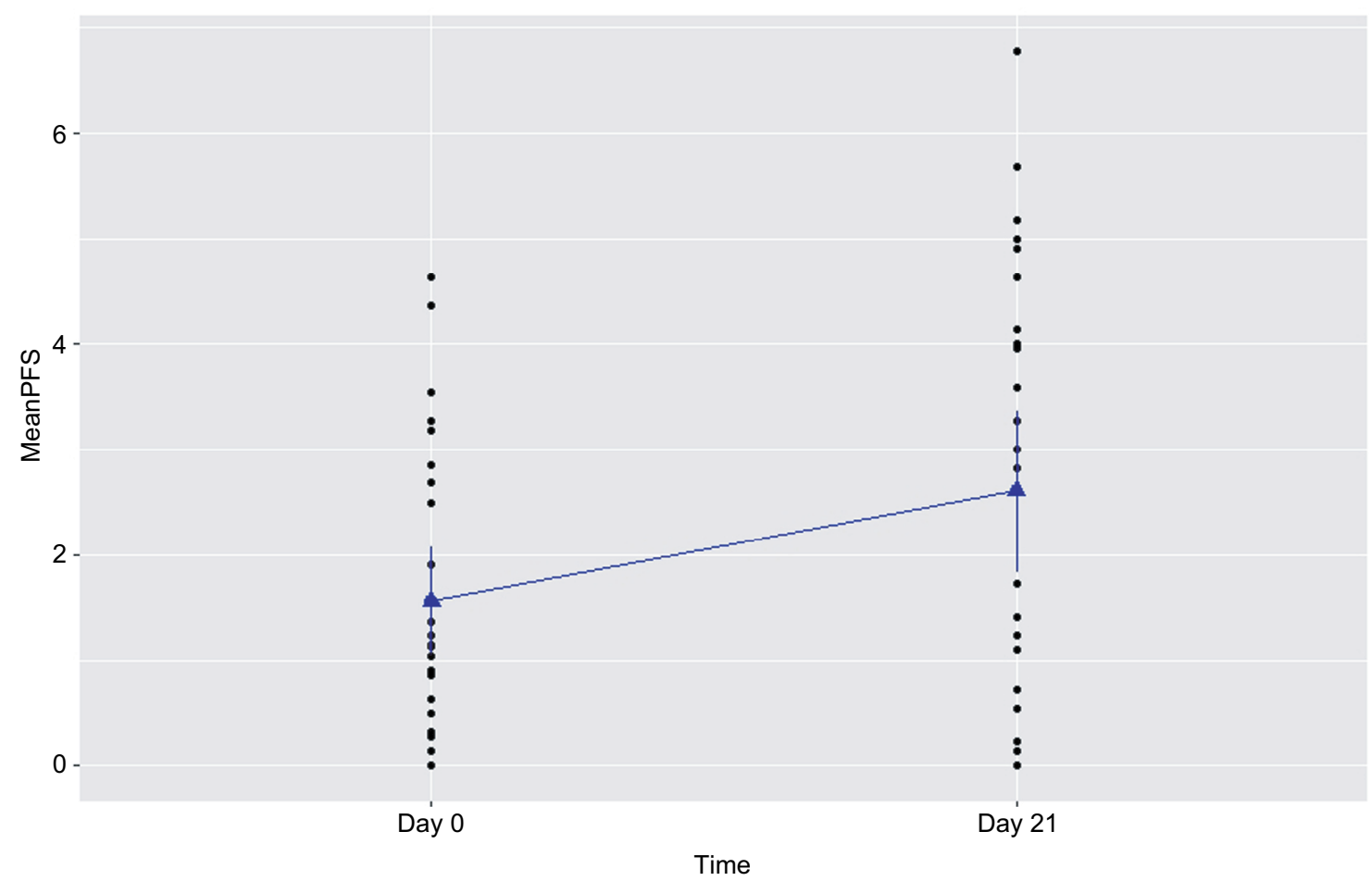

Figure 2 Increased fatigue experienced by prostate cancer patients undergoing radiation therapy $(n=15)$.

Notes: Fatigue was measured by the revised Piper Fatigue Scale (PFS) at day 0 prior to radiation therapy and day 21 of radiation therapy in patients with prostate cancer. Fatigue score was significantly increased at day 21 of radiotherapy $(P=0.003)$.

as ADP-stimulated respiration with duroquinol (DHQ), a reduced $\mathrm{CoQ}$ analog, which is a substrate for complex III. Following the addition of the uncoupler, FCCP, uncoupled complex III respiration was determined. Lastly, antimycin A was added to inhibit complex III; the antimycin A-insensitive rate was considered to be a non-mitochondrial rate.

In addition, the reserve capacity of mononuclear cell mitochondria was calculated as the difference between OXPHOS-coupled oxidation rates (complex I-linked, complex I+II-linked respiration rate) and maximal oxidative capacity-uncoupled rates (uncoupled complex I, complex II, and complex I+II) in protocol 1, and in protocol 2 as the difference between the rate of OXPHOS (coupled complex III-linked) and uncoupled complex III. In this preliminary report, we presented raw data of mitochondrial OXPHOS without the subtraction of the non-mitochondrial rate. The mitochondrial OXPHOS experiment was performed consistently by the same study investigator in the mornings. The mitochondrial OXPHOS data were normalized by the number of live peripheral blood mononuclear cells.

\section{Statistical analyses}

Descriptive statistics were calculated for the mean, standard deviation, standard error of the mean, and range for the participants' demographic, clinical characteristics, and mitochondrial OXPHOS. The paired $t$-test was used to compare the mean differences of fatigue score and mitochondrial OXPHOS in patients undergoing XRT between day 0 and day 21. General linear model was used to estimate the individual growth curve of fatigue scores and mitochondrial oxidative phosphorylation. Power analysis was employed to determine sample size of 25 to achieve $80 \%$ power with the detection of difference in fatigue and mitochondrial OXPHOS within subjects. All statistical analysis and graphics were conducted using SPSS 24.0 (IBM Corporation, Armonk, NY, USA), and R 3.0.0 for Windows. Statistical significance was defined as $P<0.05$.

\section{Results}

Fifteen males with localized prostate cancer undergoing radiation therapy were enrolled in the study between December 2015 and December 2017. The demographics and clinical characteristics of participants are described in Table 1. The average age of patients was $67.7 \pm 2.1$ years. More than half of the subjects $(53 \%, \mathrm{n}=8 / 15)$ were Caucasian, married $(53 \%, n=8 / 15)$, and retired $(60 \%, n=9 / 15)$. Most of the subjects $(87 \%, n=13 / 15)$ had a clinical stage T1c with a Gleason score of 6 or 7 in these men, the tumor was found 
Table I Patients' demographics and clinical characteristics $(n=15)$

\begin{tabular}{|c|c|}
\hline & $\begin{array}{l}\text { Mean }( \pm \text { SEM) } \\
\text { or } n(\%)\end{array}$ \\
\hline Age (years) & $67.7( \pm 2.1)$ \\
\hline \multicolumn{2}{|l|}{ Race } \\
\hline Caucasian & $8(52)$ \\
\hline African-American & $7(47)$ \\
\hline \multicolumn{2}{|l|}{ Marital status } \\
\hline Married & $8(53)$ \\
\hline Widowed & $5(33)$ \\
\hline Single & $2(14)$ \\
\hline \multicolumn{2}{|l|}{ Employment status } \\
\hline Employed full time & $5(33)$ \\
\hline Employed part time & $\mathrm{I}(7)$ \\
\hline Retired & $9(60)$ \\
\hline \multicolumn{2}{|l|}{ Clinical T stage } \\
\hline TIc & $13(87)$ \\
\hline $\mathrm{T} 2$ & $2(13)$ \\
\hline Gleason score & $7.5( \pm 0.8)$ \\
\hline Total dosage of radiation therapy (Gray) & $89.1( \pm 13.0)$ \\
\hline Karnofsky score & $88.7( \pm 1.3)$ \\
\hline \multicolumn{2}{|l|}{ PSA (ng/mL) } \\
\hline Day 0 & $12.03( \pm 4.0)$ \\
\hline \multicolumn{2}{|l|}{$\mathrm{Hb}(\mathrm{gm} / \mathrm{dL})$} \\
\hline Day 0 & $14.0 \mid( \pm 0.3)$ \\
\hline Day 21 & $13.68( \pm 0.5)$ \\
\hline \multicolumn{2}{|l|}{ Depression (HAM-D) } \\
\hline Day 0 & $1.6( \pm 0.4)$ \\
\hline Day 21 & $\mathrm{I} .8( \pm 0.4)$ \\
\hline \multicolumn{2}{|l|}{ Fatigue (r-PFS) } \\
\hline Day 0 & $1.5 \mathrm{I}( \pm 0.4)$ \\
\hline Day 21 & $3.33( \pm 0.5)$ \\
\hline
\end{tabular}

Notes: T, tumor; TI, the tumor cannot be felt during a DRE and is not seen during imaging tests. It may be found when surgery is done for another reason, usually for $\mathrm{BPH}$ or an abnormal growth of noncancerous prostate cells. TIc $=$ the tumor is found during a needle biopsy, usually because the patient has an elevated PSA level. $\mathrm{T} 2$ = the tumor is found only in the prostate, not other parts of the body. It is large enough to be felt during a DRE.

Abbreviations: $\mathrm{BPH}$, benign prostatic hyperplasia; DRE, digital rectum examination; HAM-D, Hamilton Depression Scale; PSA, prostate specific antigen; r-PFS, revised Piper Fatigue Scale; SEM, standard error of mean.

by a needle biopsy, was not palpable, and was moderately or intermediately differentiated with an elevated PSA level. ${ }^{1}$ The mean of hemoglobin levels at day 0 was $14.01 \pm 0.3$ and $13.68 \pm 0.5 \mathrm{gm}$ at day 21 of XRT. All participants scored 90 on the Karnofsky Performance Scale, indicating that they were able to carry out normal activities with only minor signs or symptoms of disease. None of the 15 participants reached the cutoff score for clinical depression $($ mean $=0.85 \pm 1.3){ }^{37}$

\section{Fatigue}

A significant increased fatigue score was reported by prostate cancer patients at day 21 of XRT, as presented in Table 1 and Figures 1 and 2. Compared to day $0(1.51 \pm 0.4)$ prior to treatment, the r-PFS scores for XRT subjects worsened significantly at day 21 of XRT $(3.33 \pm 0.5, P=0.003)$, indicating intensification of fatigue during XRT (Figure 3). While the relationship was not significant, the increased fatigue appeared to be associated with decreased OXPHOS-complex III-linked oxidation rate $(r=0.3 ; P=0.2)$ and uncoupled complex III activity $(r=0.3 ; P=0.1)$ at day 21 of XRT.

\section{Integrated mitochondrial function}

Integrated mitochondrial OXPHOS data are presented in Table 2. Intact cellular rates were not significantly different between protocol 1 and protocol 2, at day 0 and day 21 of XRT. In protocol 1, with the addition of malate and pyruvate, intact cellular respiration rate was slightly increased. OXPHOS complex I-linked rate following the addition of glutamate was not significantly different compared to that without glutamate for both day 0 and day 21 . With the addition of succinate, the substrate for complex II, the oxidation rate of OXPHOS complex I+II-linked increased to $17.55 \pm 1.73$ $\mathrm{pmol} / \mathrm{sec} / 10^{6}$ cells at day 0 and $13.55 \pm 0.88 \mathrm{pmol} / \mathrm{sec} / 10^{6} \mathrm{cells}$ at day 21 , defined as the convergence of complex I and II on coenzyme Q (CoQ). ${ }^{38}$ Compared to OXPHOS-complex I+II-linked oxidation rate, FCCP-induced maximal oxidative capacity was significantly increased $(19.78 \pm 2.03 \mathrm{pmol} /$ sec $/ 10^{6}$ cells, day $0 ; 16.19+1.04 \mathrm{pmol} / \mathrm{sec} / 10^{6}$ cells, day 21$)$. The difference between OXPHOS-complex I+II-linked respiration rate and maximal oxidation capacity was $2.23 \pm 0.16$ $\mathrm{pmol} / \mathrm{sec} / 10^{6}$ cells at day 0 and $2.64 \pm 0.21 \mathrm{pmol} / \mathrm{sec} / 10^{6} \mathrm{cells}$ at day 21 , indicating a significant but small reserve capacity for oxidation (Table 2).

The rotenone-sensitive uncoupled complex I activity was $7.62 \pm 1.9 \mathrm{pmol} / \mathrm{sec} / 10^{6}$ cells at day 0 and $6.83 \pm 0.1 \mathrm{pmol} /$ sec $/ 10^{6}$ cells at day 21 . The antimycin A-insensitive rate, considered residual oxygen consumption (non-mitochondria rate), was not different between day 0 and day 21 . Uncoupled complex II oxidation rate was $11.54 \pm 1.31 \mathrm{pmol} / \mathrm{sec} / 10^{6}$ cells at day 0 and $8.9+0.71 \mathrm{pmol} / \mathrm{sec} / 10^{6}$ cells at day 21 . At day 0 , the azide-sensitive uncoupled complex IV rate was $209.43 \pm 25.88 \mathrm{pmol} / \mathrm{sec} / 10^{6}$ cells and $205.36 \pm 25.14 \mathrm{pmol} /$ $\mathrm{sec} / 10^{6}$ cells at day 21 .

In protocol 2, we measured fatty acid OXPHOS and complex III oxidation. Intact cellular respiration rate with the addition of malate and palmitoylcarnitine was not significantly different between day 0 and day 21 of XRT. Similarly, the ADP-stimulated OXPHOS of fatty acid oxidation was not significantly different between day 0 and day 21 of XRT. Coupled complex III-linked respiration rate with DHQ as substrate was $24.63 \pm 2.61 \mathrm{pmol} / \mathrm{sec} / 10^{6}$ cells at day 


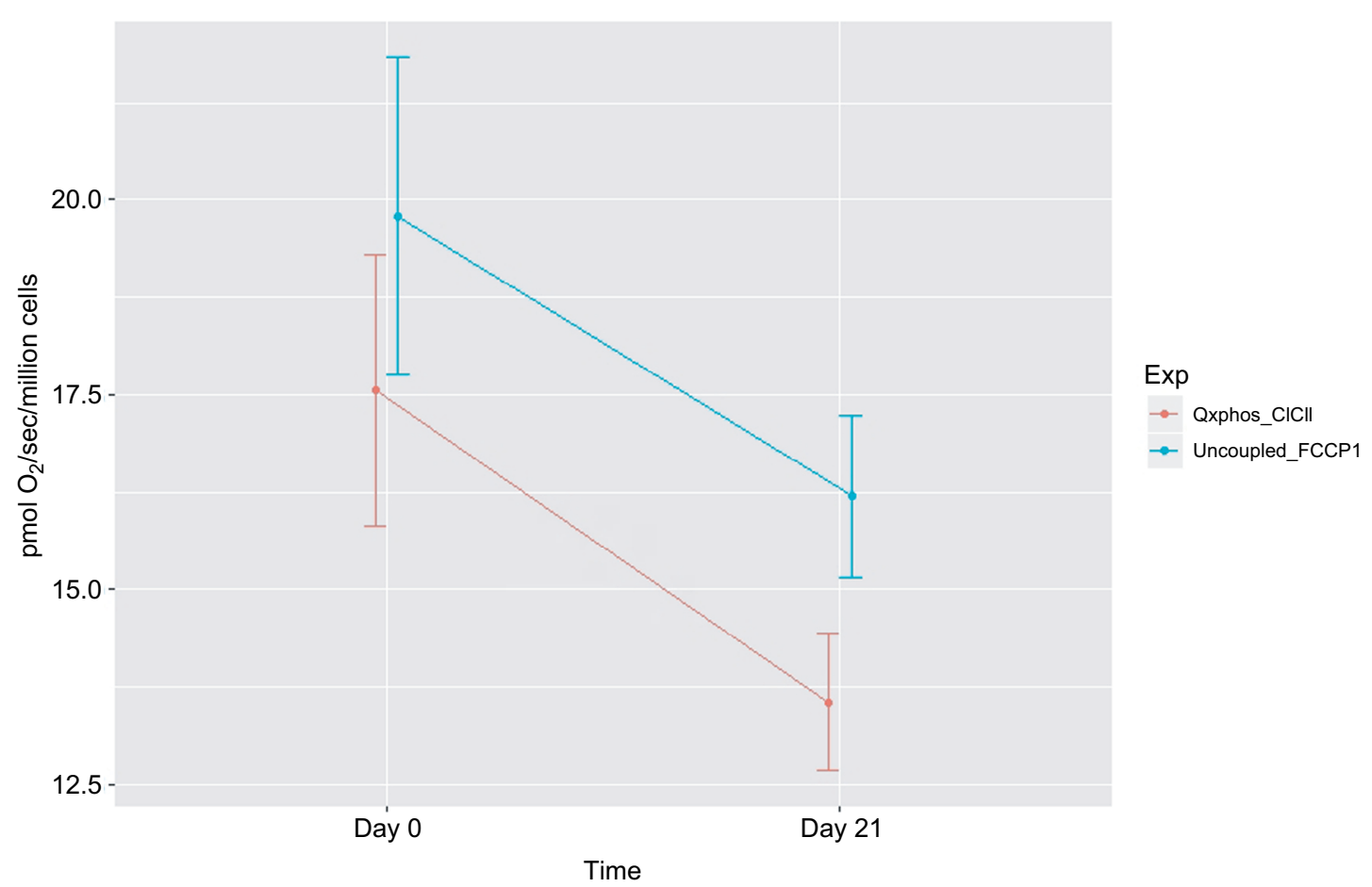

Figure 3 Decreased mitochondrial oxidative phosphorylation-OXPHOS complex I+II-linked respiration rate and maximal oxidative capacity in peripheral blood mononuclear cells of prostate cancer patients undergoing radiation therapy $(n=15)$.

Notes: The $\mathrm{X}$-axis indicates time points - day 0 (prior to radiation therapy) and day $2 \mathrm{I}$ (during radiation therapy); the $\mathrm{Y}$-axis represents cell respiration rate (pmol $\mathrm{O}_{2} /$ sec/ $10^{6}$ cells). OXPHOS was measured as the ADP-stimulated respiration with malate, pyruvate, glutamate, and succinate as substrates, reflecting the convergence of complex I and II. OXPHOS-complex I+II-linked oxidation rate was significantly decreased at day $2 \mathrm{I}$ of radiation therapy $\left(\mathrm{I} 3.55 \mathrm{pmol} \mathrm{O}_{2} / \mathrm{sec} / \mathrm{I} 0^{6} \mathrm{cells}, \mathrm{P}=0.05\right)$ from day 0 of treatment $(\mathrm{I} 7.55$ pmol $\mathrm{O}_{2} / \mathrm{sec} / 10^{6}$ cells). Uncoupled_FCCPI, uncoupled respiration rate - the maximal oxidative capacity was measured after the addition of an uncoupler-FCCP. Maximal uncoupled oxidation rate was decreased at day $2 \mathrm{I}$ of $X R T\left(I 8.9 \mathrm{pmol} \mathrm{O}_{2} / \mathrm{sec} / 10^{6} \mathrm{cells}, \mathrm{P}=0.057\right)$ compared to prior treatment ( $\left.16.3 \mathrm{pmol} \mathrm{O}_{2} / \mathrm{sec} / 10^{6} \mathrm{cells}\right)$. Abbreviations: ADP, adenosine diphosphate; FCCP, carbonylcyanide-p-trifluoromethoxyphenylhydrazone; OXPHOS, oxidative phosphorylation; XRT, radiation therapy.

21 of XRT, a decrease of $3.99 \mathrm{pmol} / \mathrm{sec} / 10^{6}$ cells from that compared to day 0 . Moreover, uncoupled complex III oxidation rate with the addition of FCCP was $25.64 \pm 2.72 \mathrm{pmol} /$ $\mathrm{sec} / 10^{6}$ cells at day 21 of XRT, a decrease of $4.06 \mathrm{pmol} / \mathrm{sec} / 10^{6}$ cells from that at day 0 . The difference of oxidation rates between coupled complex III-linked and uncoupled complex III was $1.08 \pm 0.41 \mathrm{pmol} / \mathrm{sec} / 10^{6}$ cells at day 0 and $1.01 \pm 0.33$ $\mathrm{pmol} / \mathrm{sec} / 10^{6}$ cells at day $21(P=0.06)$, reflecting little to no reserve capacity of mononuclear cell mitochondria starting at complex III (Table 2).

Compared to mitochondrial OXPHOS data ahead of radiation therapy, a significant decrease of OXPHOS complex I+II-linked rate $\left(\downarrow 4 \mathrm{pmol} / \mathrm{sec} / 10^{6}\right.$ cells, Figure 4$)$ and decreased maximal oxidative capacity $(\downarrow 3.59 \mathrm{pmol} /$ sec $/ 10^{6}$ cells, $P=0.057$; Figure 4$)$ were observed at day 21 of XRT. Additionally, uncoupled complex II oxidation was significantly decreased $\left(\downarrow 2.64 \mathrm{pmol} / \mathrm{sec} / 10^{6}\right.$ cells, Table 2$)$ in mononuclear cells of patients with prostate cancer at day 21 of XRT. Furthermore, coupled complex III-linked oxidation rate and uncoupled complex III activity were significantly decreased in mononuclear cell mitochondria of prostate cancer patients during XRT $\downarrow 3.99 \mathrm{pmol} / \mathrm{sec} / 10^{6}$ cells, $\downarrow 4.06$ $\mathrm{pmol} / \mathrm{sec} / 10^{6} \mathrm{cells}$, respectively (Figure 5 and Table 2).

\section{Discussion}

This preliminary evidence is the first to our knowledge to describe changes of integrated mitochondrial function in peripheral blood mononuclear cells from patients with prostate cancer undergoing XRT. Our major findings include: 1) Prostate cancer patients experienced intensification of fatigue at day 21 during XRT; and 2) A defect in oxidation starting at complex III in mononuclear cell mitochondria of patients with prostate cancer was identified at day 21 during XRT.

The trajectory of fatigue reported in this study is consistent with other studies ${ }^{5,12}$ and our previous findings. ${ }^{22,24,25}$ This progression suggests that biologic mechanisms within mitochondria related to the repeated stress imposed by daily doses of irradiation are likely involved in the worsening of fatigue symptoms in this population. Further, the trajectory 
Table 2 Integrated mitochondrial function in peripheral blood mononuclear cells from prostate cancer patients undergoing radiation therapy $(n=15)$

\begin{tabular}{|c|c|c|c|}
\hline \multirow{2}{*}{$\begin{array}{l}\text { Indicators of integrated mitochondrial function } \\
\text { pmol } \mathrm{O}_{2} / \mathrm{sec} / 10^{6} \text { cells }\end{array}$} & \multicolumn{3}{|l|}{ Mean \pm SEM } \\
\hline & $\begin{array}{l}\text { Day } 0 \text { (prior } \\
\text { radiotherapy) }\end{array}$ & $\begin{array}{l}\text { Day } 2 \text { I (during } \\
\text { radiotherapy) }\end{array}$ & $\begin{array}{l}\text { Difference between } \\
\text { day } 0 \text { and day } 21\end{array}$ \\
\hline \multicolumn{4}{|l|}{ Protocol I } \\
\hline Intact cell respiration & $6.96 \pm 0.41$ & $5.65 \pm 0.5 \mathrm{I}$ & $\downarrow I .31$ \\
\hline Intact cell respiration $+(m+p)$ & $7.59 \pm 0.52$ & $6.8 I \pm 0.64$ & $\downarrow 0.78$ \\
\hline OXPHOS (Cl-linked) & $7.61 \pm 0.92$ & $6.93 \pm 0.66$ & $\downarrow 0.68$ \\
\hline OXPHOS (Cl + Cll-linked) & $17.55 \pm 1.73$ & $13.55 \pm 0.88$ & $\downarrow 4.0^{*}$ \\
\hline Maximal oxidative capacity (uncoupler, FCCP) & $19.78 \pm 2.03$ & $16.19 \pm 1.04$ & $\downarrow 3.59$ \\
\hline Uncoupled $\mathrm{Cl}$ & $7.62 \pm 1.91$ & $6.83 \pm 1.72$ & $\downarrow 0.79$ \\
\hline Uncoupled Cll & $11.54 \pm 1.31$ & $8.9 \pm 0.71$ & $\downarrow 2.64^{*}$ \\
\hline Uncoupled CIV & $209.43 \pm 25.88$ & $205.36 \pm 25.14$ & $\downarrow 4.1$ \\
\hline $\begin{array}{l}\text { Reserve capacity } \\
\text { (Difference between OXPHOS }[\mathrm{Cl}+\mathrm{Cll}-\text { linked }] \text { and maximal oxidative } \\
\text { capacity) }\end{array}$ & $2.23 \pm 0.16 *$ & $2.64 \pm 0.2 I^{*}$ & $\uparrow 0.4 \mathrm{I}$ \\
\hline $\begin{array}{l}\text { Citrate synthase } \\
\left(\mathrm{nmol} / \mathrm{min} / \mathrm{mg} / 10^{6} \text { cells }\right)\end{array}$ & $3.91 \pm 1.05$ & $3.12 \pm 0.51$ & $\downarrow 0.79$ \\
\hline \multicolumn{4}{|l|}{ Protocol 2} \\
\hline Intact cell respiration & $6.21 \pm 0.48$ & $6.7 I \pm 0.89$ & $\uparrow 0.50$ \\
\hline Intact cell respiration $+(m+p a l)$ & $6.34 \pm 0.59$ & $7.02 \pm 1.01$ & $\uparrow 0.68$ \\
\hline OXPHOS (fatty acid oxidation) & $5.35 \pm 0.65$ & $6.75 \pm 0.91$ & $\uparrow \mathrm{I} .4$ \\
\hline OXPHOS (coupled CIII; DHQ) & $28.62 \pm 2.92$ & $24.63 \pm 2.61$ & $\downarrow 3.99 *$ \\
\hline Uncoupled Complex III (uncoupler, FCCP) & $29.7 \pm 3.21$ & $25.64 \pm 2.72$ & $\downarrow 4.06^{*}$ \\
\hline $\begin{array}{l}\text { Reserve capacity } \\
\text { (Difference between OXPHOS [coupled CIII-linked; DHQ] and uncoupled } \\
\text { complex III }\end{array}$ & $I .08 \pm 0.4 \mathrm{I}$ & $1.01 \pm 0.33$ & $\downarrow 0.07$ \\
\hline $\begin{array}{l}\text { Citrate synthase } \\
\left(\mathrm{nmol} / \mathrm{min} / \mathrm{mg} / 10^{6} \text { cells }\right)\end{array}$ & $4.94 \pm 1.26$ & $5.75 \pm 1.17$ & $\uparrow 0.8 \mathrm{I}$ \\
\hline
\end{tabular}

Notes: In protocol I, intact cell respiration $+(\mathrm{m}+\mathrm{p})$ was measured after the addition of malate and pyruvate before permeabilization; in protocol 2 , intact cell respiration + $(\mathrm{m}+\mathrm{pal})$ was determined after the addition of malate and palmitoylcarnitine before permeabilization. Data are presented as mean \pm standard error of mean. $* P<0.05$.

Abbreviations: Cl, complex I; CII, complex II; CIII, complex III; CIV, complex IV; DHQ, duroquinol; FCCP, carbonylcyanide-p-trifluoromethoxyphenylhydrazone; m, malate; OXPHOS, oxidative phosphorylation; p, pyruvate; pal, palmitoylcarnitine; sec, second; SEM, standard error of mean.

of fatigue intensification, as an acute effect of XRT, suggests that implementing interventions for XRT-associated fatigue should start early.

A defect in mononuclear cell mitochondrial complex III oxidation was revealed in patients with prostate cancer at day 21 of XRT. Comparing changes in oxidation rates of coupled III and uncoupled complex III between day 0 and day 21 of XRT, significantly decreased coupled complex III rate $(\downarrow 13.9 \%)$, and uncoupled CIII activity $(\downarrow 13.7 \%)$ were detected in mononuclear cell mitochondria at day 21 of XRT in prostate cancer patients. At day 21 of XRT, uncoupled complex III oxidation rate was not completely relieved by uncoupling and remained low $\left(25.64 \mathrm{pmol} / \mathrm{sec} / 10^{6}\right.$ cells $)$ compared to that at day $0\left(29.7 \mathrm{pmol} / \mathrm{sec} / 10^{6}\right.$ cells $)$. In addition, the uncoupled complex IV oxidation rate was similar at day 21 of XRT to that at day 0 of XRT in patients with prostate cancer. This indicates a defect in oxidation starting at complex III in mononuclear cell mitochondria of patients with prostate cancer at day 21 during XRT.

In prostate cancer patients' mononuclear cell mitochondria, we documented significant decreases of OXPHOScomplex I+II-linked respiration $(\downarrow 22.8 \%)$ and maximal oxidative capacity-uncoupled complex I+II $(\downarrow 18.1 \%)$ at day 21 of XRT compared to that at day 0 of XRT. There was no change of oxidation rate for coupled complex I and uncoupled complex I at day 21 of XRT compared to that at day 0 of XRT in patients with prostate cancer. Additionally, maximal oxidative capacity, uncoupled oxidation, starting at complex II was significantly decreased $(\downarrow 22.9 \%)$ in mononuclear cell mitochondria at day 21 of XRT compared to that at day 0 before XRT. Using peripheral blood mononuclear cells, other investigators have reported decreased mitochondrial OXPHOS complex I and II-linked oxidation rates associated with the symptom of depression and fatigue in patients with 


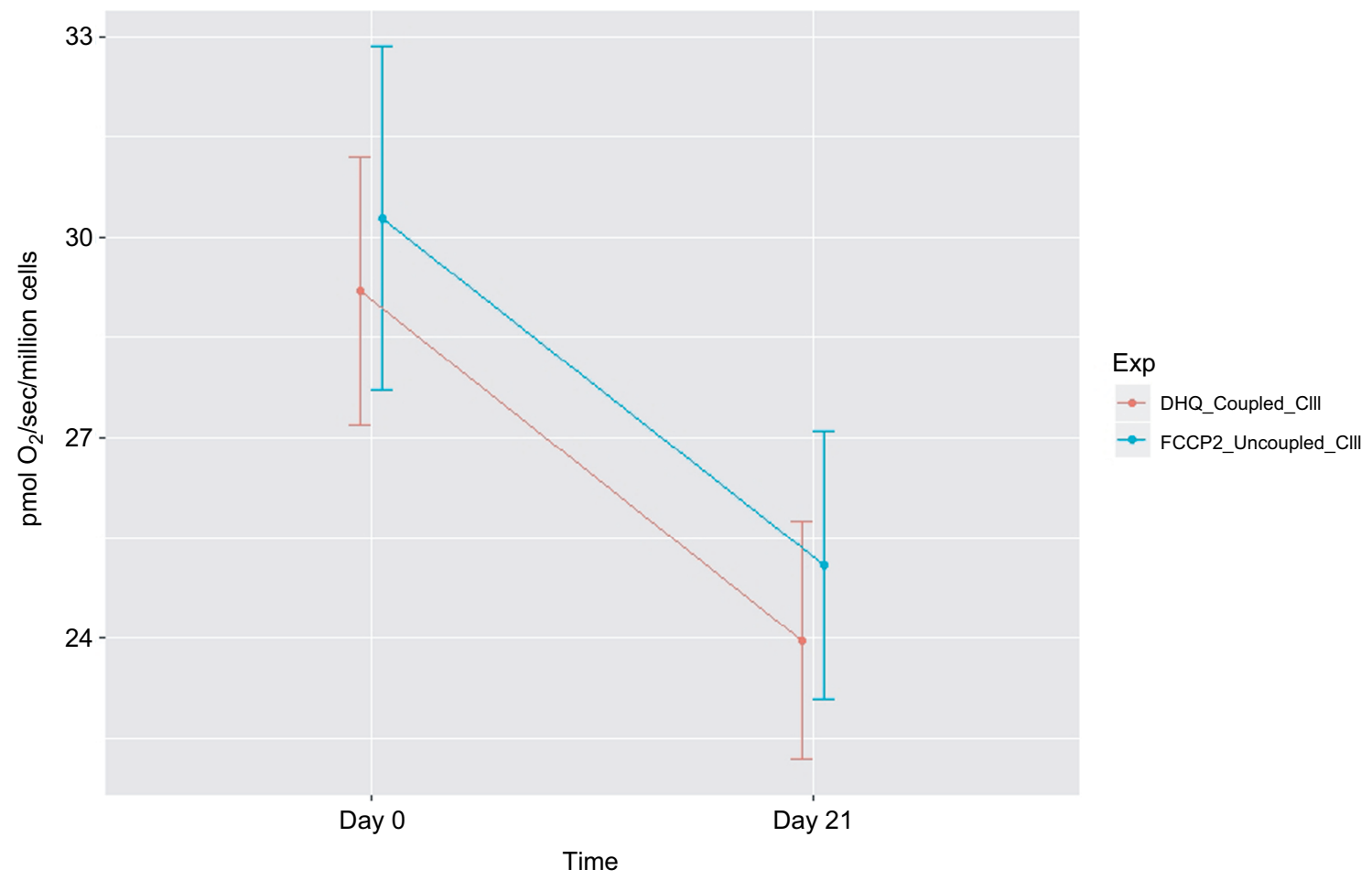

Figure 4 Decreased mitochondrial oxidative phosphorylation-OXPHOS complex III-linked and uncoupled complex III oxidation rate in peripheral blood mononuclear cells of prostate cancer patients undergoing radiation therapy $(n=15)$.

Notes: The $\mathrm{X}$-axis indicates time points - day 0 (prior radiation therapy) and day $2 \mathrm{I}$ (during radiation therapy); the $\mathrm{Y}$-axis represents cell respiration rate (pmol $\mathrm{O}_{2} /$ sec $/ 10^{6}$ cells); OXPHOS-complex III-linked, coupled complex III rate was measured as the ADP-stimulated respiration with DHQ, a substrate for complex III, representing respiration rate of coupled complex III. Uncoupled complex III was determined after the addition of an uncoupler, FCCP. Both coupled and uncoupled complexes III oxidation rates were significantly decreased at day $2 \mathrm{I}$ of $\mathrm{XRT}$ from day $0(P<0.05)$.

Abbreviations: ADP, adenosine diphosphate; DHQ, duroquinol; FCCP, carbonylcyanide-p-trifluoromethoxyphenylhydrazone; OXPHOS, oxidative phosphorylation; XRT, radiation therapy.

major depression, ${ }^{39}$ in patients with Huntington disease, ${ }^{40,41}$ and in pediatric septic shock..$^{42}$ These studies did not evaluate the OXPHOS-complex III-linked oxidation rate in human peripheral blood mononuclear cell mitochondria.

Mitochondrial complex I and II catalyze electron transfer to ubiquinone from two different donors: NADH (complex I) and succinate (complex II). Complex III carries electrons from reduced ubiquinone to cytochrome $c$, and complex IV completes the sequence by transferring electrons from cytochrome $c$ to $\mathrm{O}_{2}{ }^{43}$ The defect in oxidation starting at complex III affected upstream complex II oxidation, but not complex I or fatty acid oxidation. Based on our preliminary finding, we propose that the defect in complex III was not severe enough to limit complex I or fatty acid oxidation, but sufficient to affect the activity of complex II-uncoupled oxidation.

In addition, we evaluated whether OXPHOS was limited by the oxidation or the phosphorylation component. The OXPHOS-coupled oxidation rate and maximal oxidative capacity-uncoupled activity, coupled complex I-linked activity was identical to uncoupled complex I activity reflecting the limiting step for OXPHOS starting at complex I was oxidation. Uncoupled complex II oxidation was increased compared to coupled complex II-linked activity, indicating that the limiting step in OXPHOS starting at complex II was phosphorylation (Table 2). For complex III, there was no significant difference of oxidation rates between coupled and uncoupled at both day 0 and day 21 of XRT, pointing to the limiting step for OXPHOS starting at complex III was oxidation with no reserve capacity in peripheral blood mononuclear cell mitochondria of patients with prostate cancer undergoing XRT. Mitochondrial reserve respiratory capacity is an important component of cell bioenergetics because it can be called upon during an increase in energy demand. ${ }^{44}$

\section{Limitations}

This preliminary report is part of an ongoing study. The analysis reported here focused on day 21 of XRT -3 weeks after the beginning of XRT; therefore, the results are limited to acute effects of radiation-associated fatigue and altered mitochondrial function and may not be reflective of changes 
that occur altering the trajectory of treatment and recovery. Another issue is the small sample size; a larger sample should be pursued to confirm the associations of altered mitochondrial integrated function with CRF for further study. An additional limitation was the lack of measure of variables that might affect $\mathrm{CRF}$ such as impaired cognitive function and sleep disturbance. Mitochondrial OXPHOS was measured using intact mitochondria, providing screening of systemic mitochondrial dysfunction; electron transport chain enzymatic activity will further assist in identifying the specific complex defects..$^{34,45}$

\section{Conclusion}

A defect in oxidation starting at complex III in mononuclear cell mitochondria is revealed at day 21 of XRT in prostate cancer patients receiving XRT. Fatigue symptoms occur in patients with prostate cancer undergoing radiotherapy; furthermore, decreased complex III oxidation appears to be associated with increased fatigue symptom in prostate cancer patients at day 21 of XRT. We suggest that complex III plays a role in developing radiation-induced fatigue. Complex III is a potential target for pharmacological and, in particular, nutraceutical interventions; for example, coenzyme $\mathrm{Q}$ and ascorbate to bypass complex III might improve radiationinduced fatigue.

\section{Acknowledgments}

This study was supported by the National Institute of Nursing Research, National Institutes of Health (K01 NR015246, 2015-2018); the Oncology Nursing Society Foundation, Pittsburg, PA (RES125833, 2014-2015); and the Center for Mitochondrial Disease, Case Western Reserve University. We appreciate the support and guidance of Dr Rodney Ellis, and the editing assistance of English language and grammar by Bernard Tandler, PhD.

\section{Disclosure}

The authors report no conflicts of interest in this work.

\section{References}

1. American Cancer Society. Cancer Facts \& Figures 2018-American Cancer Society; 2018. Available from: https://www.cancer.org/content/ $\mathrm{dam} /$ cancer-org/research/cancer-facts-and-statistics/annual-cancerfacts-and-figures/2018/cancer-facts-and-figures-2018.pdf. Accessed November 21, 2018.

2. Siegel RL, Miller KD, Jemal A. Cancer statistics, 2018. CA Cancer J Clin. 2018;68(1):7-30.

3. Pinkawa M, Gontero P. Open to debate. The motion: radiotherapy for prostate cancer preserves sexual function to a greater extent than nerve sparing radical prostatectomy. Eur Urol. 2009;56(1):212-214.

4. Damber JE, Aus G. Prostate cancer. Lancet. 2008;371(9625):1710-1721.
5. Langston B, Armes J, Levy A, Tidey E, Ream E. The prevalence and severity of fatigue in men with prostate cancer: a systematic review of the literature. Support Care Cancer. 2013;21(6):1761-1771.

6. Brix C, Schleussner C, Füller J, Röhrig B, Strauss B. Fatigue and its determinants in radio-oncology. Psychother Psychosom Med Psychol. 2009;59(2):42-49.

7. Lardas M, Liew M, van den Bergh RC, et al. Quality of life outcomes after primary treatment for clinically localised prostate cancer: a systematic review. Eur Urol. 2017;72(6):869-885.

8. Wang XS, Zhao F, Fisch MJ, et al. Prevalence and characteristics of moderate to severe fatigue: a multicenter study in cancer patients and survivors. Cancer. 2014;120(3):425-432.

9. Wang XS. Pathophysiology of cancer-related fatigue. Clin J Oncol Nurs. 2008;12(5):11-20.

10. Minton O, Berger A, Barsevick A, et al. Cancer-related fatigue and its impact on functioning. Cancer. 2013;119(S11):2124-2130.

11. Shaitelman SF, Schlembach PJ, Arzu I, et al. Acute and short-term toxic effects of conventionally fractionated vs hypofractionated whole-breast irradiation: A randomized clinical trial. JAMA Oncol. 2015;1(7):931-941.

12. Miaskowski C, Paul SM, Cooper BA, et al. Trajectories of fatigue in men with prostate cancer before, during, and after radiation therapy. J Pain Symptom Manage. 2008;35(6):632-643.

13. National Comprehensive Cancer Network. Clinical Practice Guidelines in Oncology. Cancer-Related Fatigue. Version 2. February 20, 2018. Available from: https://www.nccn.org/professionals/physician_gls/pdf/ fatigue.pdf. Accessed November 21, 2018.

14. Blackhall L, Petroni G, Shu J, Baum L, Farace E. A pilot study evaluating the safety and efficacy of modafinal for cancer-related fatigue. J Palliat Med. 2009;12(5):433-439.

15. Byar KL, Berger AM, Bakken SL, Cetak MA. Impact of adjuvant breast cancer chemotherapy on fatigue, other symptoms, and quality of life. Oncol Nurs Forum. 2006;33(1):E18-E26.

16. Berger AM, Mitchell SA. Modifying cancer-related fatigue by optimizing sleep quality. J Natl Compr Canc Netw. 2008;6(1):3-13.

17. Monga U, Kerrigan AJ, Thornby J, Monga TN. Prospective study of fatigue in localized prostate cancer patients undergoing radiotherapy. Radiat Oncol Investig. 1999;7(3):178-185.

18. St Pierre BA, Kasper CE, Lindsey AM. Fatigue mechanisms in patients with cancer: effects of tumor necrosis factor and exercise on skeletal muscle. Oncol Nurs Forum. 1992;19(3):419-425.

19. Monga U, Jaweed M, Kerrigan AJ, et al. Neuromuscular fatigue in prostate cancer patients undergoing radiation therapy. Arch Phys Med Rehabil. 1997;78(9):961-966.

20. Ryan JL, Carroll JK, Ryan EP, Mustian KM, Fiscella K, Morrow GR. Mechanisms of cancer-related fatigue. Oncologist. 2007;12(Suppl 1):22-34.

21. Yavuzsen T, Davis MP, Ranganathan VK, et al. Cancer-related fatigue: central or peripheral? J Pain Symptom Manage. 2009;38(4):587-596.

22. Saligan LN, Hsiao CP, Wang D, et al. Upregulation of $\alpha$-synuclein during localized radiation therapy signals the association of cancerrelated fatigue with the activation of inflammatory and neuroprotective pathways. Brain Behav Immun. 2013;27(1):63-70.

23. Bruera E, Valero V, Driver L, et al. Patient-controlled methylphenidate for cancer fatigue: a double-blind, randomized, placebo-controlled trial. J Clin Oncol. 2006;24(13):2073-2078.

24. Hsiao CP, Wang D, Kaushal A, Saligan L. Mitochondria-related gene expression changes are associated with fatigue in patients with nonmetastatic prostate cancer receiving external beam radiation therapy. Cancer Nurs. 2013;36(3):189-197.

25. Hsiao CP, Wang D, Kaushal A, Chen MK, Saligan L. Differential expression of genes related to mitochondrial biogenesis and bioenergetics in fatigued prostate cancer men receiving external beam radiation therapy. J Pain Symptom Manage. 2014;48(6):1080-1090.

26. Hsiao CP, Daly B, Hoppel C. Association between mitochondrial bioenergetics and radiation- related fatigue: a possible mechanism and novel target. Arch Cancer Res. 2015;3(2):14. 
27. van den Heuvel L, Smeitink J. The oxidative phosphorylation (OXPHOS) system: nuclear genes and human genetic diseases. Bioessays. 2001;23(6):518-525.

28. Davis RE, Williams M. Mitochondrial function and dysfunction: an update. J Pharmacol Exp Ther. 2012;342(3):598-607.

29. Puchowicz MA, Varnes ME, Cohen BH, Friedman NR, Kerr DS, Hoppel CL. Oxidative phosphorylation analysis: assessing the integrated functional activity of human skeletal muscle mitochondria-case studies. Mitochondrion. 2004;4(5-6):377-385.

30. Piper BF. Piper Fatigue Scale available for clinical testing. Oncol Nurs Forum. 1990;17(5):661-662.

31. Lydiatt WM, Denman D, McNeilly DP, Puumula SE, Burke WJ. A randomized, placebo-controlled trial of citalopram for the prevention of major depression during treatment for head and neck cancer. Arch Otolaryngol Head Neck Surg. 2008;134(5):528-535.

32. Hsiao CP, Hoppel C. Analyzing mitochondrial function in human peripheral blood mononuclear cells. Anal Biochem. 2018;549: $12-20$.

33. Pesta D, Gnaiger E. High-resolution respirometry: OXPHOS protocols for human cells and permeabilized fibers from small biopsies of human muscle. Methods Mol Biol. 2012;810:25-58.

34. Puchowicz MA, Varnes ME, Cohen BH, Friedman NR, Kerr DS, Hoppel CL. Oxidative phosphorylation analysis: assessing the integrated functional activity of human skeletal muscle mitochondria - case studies Mitochondrion. 2004;4(5-6):377-385.

35. Ye F, Hoppel CL. Measuring oxidative phosphorylation in human skin fibroblasts. Anal Biochem. 2013;437(1):52-58.

36. Tsai HH, Chang SC, Chou CH, Weng TP, Hsu CC, Wang JS. Exercise training alleviates hypoxia-induced mitochondrial dysfunction in the lymphocytes of sedentary males. Sci Rep. 2016;6:35170.
37. Lazure KE, Lydiatt WM, Denman D, Burke WJ. Association between depression and survival or disease recurrence in patients with head and neck cancer enrolled in a depression prevention trial. Head Neck. 2009;31(7):888-892.

38. Gnaiger E. Mitopathways to complexes I+II. In: Gnaiger E, editor. Mitochondrial Pathways and Respiratory Control: An Introduction to OXPHOS Analysis. Innsbruck, Austria: OROBOROS; 2012:29-44.

39. Karabatsiakis A, Böck C, Salinas-Manrique J, et al. Mitochondrial respiration in peripheral blood mononuclear cells correlates with depressive subsymptoms and severity of major depression. Transl Psychiatry. 2014;4(6):e397.

40. Sjövall F, Morota S, Persson J, Hansson MJ, Elmér E. Patients with sepsis exhibit increased mitochondrial respiratory capacity in peripheral blood immune cells. Crit Care. 2013;17(4):R152.

41. Ehinger JK, Morota S, Hansson MJ, Paul G, Elmér E. Mitochondrial respiratory function in peripheral blood cells from Huntington's Disease patients. Mov Disord Clin Pract. 2016;3(5):472-482.

42. Weiss SL, Selak MA, Tuluc F, et al. Mitochondrial dysfunction in peripheral blood mononuclear cells in pediatric septic shock. Pediatr Crit Care Med. 2015;16(1):e4-e12.

43. Lenaz G, Genova ML. Structure and organization of mitochondrial respiratory complexes: a new understanding of an old subject. Antioxid Redox Signal. 2010;12(8):961-1008.

44. Pfleger J, He M, Abdellatif M. Mitochondrial complex II is a source of the reserve respiratory capacity that is regulated by metabolic sensors and promotes cell survival. Cell Death Dis. 2015;6(7):e1835.

45. Krahenbuhl S, Chang M, Brass EP, Hoppel CL. Decreased activities of ubiquinol:ferricytochrome c oxidoreductase (complex III) and ferrocytochrome c:oxygen oxidoreductase (complex IV) in liver mitochondria from rats with hydroxycobalamin[c-lactam]-induced methylmalonic aciduria. J Biol Chem. 1991;266(31):20998-21003.
Cancer Management and Research

\section{Publish your work in this journal}

Cancer Management and Research is an international, peer-reviewed open access journal focusing on cancer research and the optimal use of preventative and integrated treatment interventions to achieve improved outcomes, enhanced survival and quality of life for the cancer patient. The manuscript management system is completely online and includes

\section{Dovepress}

a very quick and fair peer-review system, which is all easy to use. Visit http://www.dovepress.com/testimonials.php to read real quotes from published authors. 